\title{
Imaging with Polarized Neutrons
}

Kardjilov, Nikolay; Hilger, Andre; Manke, Ingo; Strobl, Markus; Banhart, John

Published in:

Journal of Imaging

DOI:

10.3390/jimaging 4010023

Publication date:

2018

Document version

Publisher's PDF, also known as Version of record

Document license:

CC BY

Citation for published version (APA):

Kardjilov, N., Hilger, A., Manke, I., Strobl, M., \& Banhart, J. (2018). Imaging with Polarized Neutrons. Journal of Imaging, 4(1), [UNSP23]. https://doi.org/10.3390/jimaging4010023 


\title{
Imaging with Polarized Neutrons
}

\author{
Nikolay Kardjilov ${ }^{1, *}$, André Hilger ${ }^{1,2}$, Ingo Manke ${ }^{1}$, Markus Strobl ${ }^{3,4}$ and John Banhart ${ }^{1,2}$ \\ 1 Institute of Applied Materials, Helmholtz-Zentrum-Berlin, 14109 Berlin, Germany; \\ hilger@helmholtz-berlin.de (A.H.); manke@helmholtz-berlin.de (I.M.); banhart@helmholtz-berlin.de (J.B.) \\ 2 Technische Universität Berlin, Institut für Werkstoffwissenschaften und -technologien, 10623 Berlin, Germany \\ 3 Laboratory for Neutron Scattering and Imaging (LNS), Paul-Scherrer-Institute, 5232 Villigen, Switzerland; \\ markus.strobl@psi.ch \\ 4 Niels Bohr Institute, University of Copenhagen, 2100 Copenhagen, Denmark \\ * Correspondence: kardjilov@helmholtz-berlin.de; Tel.: +49-30-8062-42298
}

Received: 1 November 2017; Accepted: 11 January 2018; Published: 16 January 2018

\begin{abstract}
Owing to their zero charge, neutrons are able to pass through thick layers of matter (typically several centimeters) while being sensitive to magnetic fields due to their intrinsic magnetic moment. Therefore, in addition to the conventional attenuation contrast image, the magnetic field inside and around a sample can be visualized by detecting changes of polarization in a transmitted beam. The method is based on the spatially resolved measurement of the cumulative precession angles of a collimated, polarized, monochromatic neutron beam that traverses a magnetic field or sample.
\end{abstract}

Keywords: neutron imaging; polarized neutrons; flux pinning; magnetic field

\section{Introduction}

Conventional methods for imaging magnetic fields are limited to the surface and the surrounding of a sample [1]. The observation of spatial distribution of magnetic fields inside a bulk sample was not possible heretofore. In order to solve this problem, a new imaging technique utilizing polarized neutrons was proposed [2-4]. The method allows one to reveal the three-dimensional distribution of magnetic fields in solid materials.

Neutrons are subatomic particles with no electric charge, which allows them to penetrate thick layers of matter. They possess a magnetic moment making them sensitive to magnetic fields [5]. Utilizing the associated magnetic interaction for neutron imaging it is possible to visualize magnetic fields not only in free space but also in the bulk of samples.

In an applied magnetic field of strength $B$, the spin of the neutron, which is always aligned anti-parallel to its magnetic moment, will undergo Larmor precession around the field with a precession frequency $\omega_{L}=\gamma B$, where $\gamma$ is the gyromagnetic ratio of the neutron $\left(-1.832 \times 10^{8} \mathrm{rad} \mathrm{s}^{-1} \mathrm{~T}^{-1}\right)$ and $B$ is the scalar magnitude of the magnetic field. If the field has a significant component perpendicular to the magnetic moment of the neutron, precession will cause the neutrons to oscillate between the two possible spin-states (spin up and spin down states).

For a neutron traversing a magnetic field, the rate of precession is related to the strength of the field, and the total angle of precession $\varphi$ is proportional to the field strength $B$, the wavelength of the neutrons $\lambda$ and the length of the path $s$ through the field: $\varphi=\frac{\gamma \lambda m}{h} \int_{\text {path }} B d s$, where $m$ is the neutron rest mass $\left(1.675 \times 10^{-27} \mathrm{~kg}\right)$ and $h$ is Planck's constant $\left(6.626 \times 10^{-34} \mathrm{~J} \mathrm{~s}^{-1}\right)$ [6]. Thus, for a monochromatic beam, variations in the total precession angle for a given path through a magnetic field are indicative of the distribution of the field along that path $[7,8]$. These variations can be measured using a polarized beam, i.e., one in which all neutrons of one of the two possible spin-states have been removed from the beam. The polarization vector, $P$, may be considered as the average spin orientation over the 
whole beam and it is possible to consider precession of the polarization rather than of each individual neutron spin.

\section{Experimental Techniques}

\subsection{Solid-State Benders}

Magnetic imaging experiments with polarized neutrons have recently been undertaken at various imaging facilities. The first experiments were performed with a polarizer-analyzer arrangement based on solid state benders [2]. These benders consist of thin plates, usually Si wafers, which are covered on one side by a super mirror layer reflecting neutrons according to classical optics principles [9].

Applying strong magnetic fields of several tenths $\mathrm{mT}$ perpendicular to the propagation direction of the neutrons, different refractive indices for spin-up and -down neutrons occur and cause a different deflection of the two spin states, thus giving rise to two sub-beams possessing different directions. By using a collimator or by covering the non-reflecting surface of the substrates by a strongly absorbing layer (e.g., Gd) one spin component can be selected. In this way, a setup for magnetic imaging is obtained in which a solid-state bender is used to polarize a neutron beam, which is then directed through magnetic samples in which precession sets in before the beam is analyzed with a second bender [3], Figure 1a. The key to image formation is that the analyzer only transmits the polarization component that is parallel to its own polarization axis. As a result, the intensity measured behind the analyzer $I$ is the initial beam intensity $I_{0}$ decreased by conventional attenuation with attenuation coefficient $\Sigma$ (the magnitude of which can be found from a standard radiograph) and modulated by some sinusoidal function that is directly related to the angle $\phi$ by which the polarization has precessed: $I=I_{0} \exp \left(-\int_{\text {path }} \Sigma(s) d s\right) \frac{1}{2}(1+\cos (\varphi))$. Maximum (minimum) intensity will be measured whenever beam polarization and the analyzer are aligned perfectly in parallel (anti-parallel).

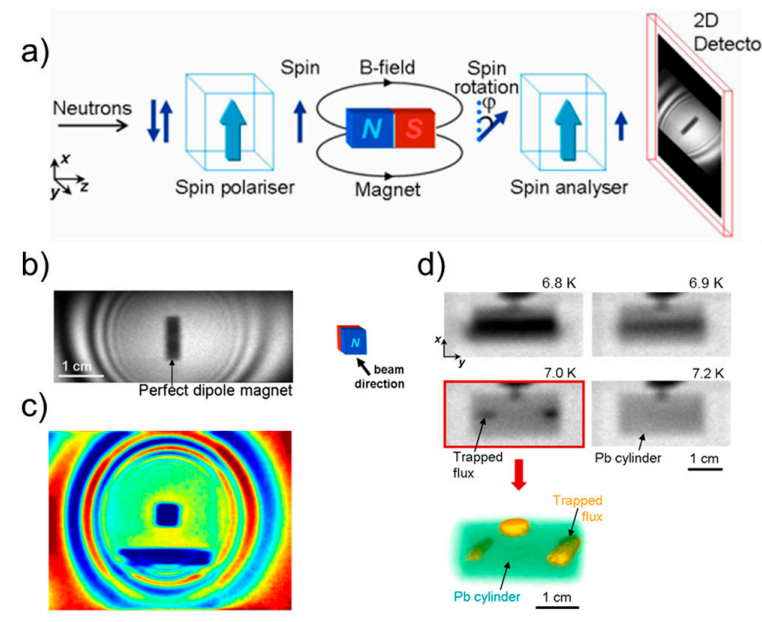

Figure 1. (a) Schematic diagram of a set-up used for imaging magnetic materials and fields. The neutron beam is polarized, then precesses in a magnetic field, eventually is analyzed and detected. Note that the intensity (dark blue arrow) behind the analyzer is smaller than the intensity behind the polarizer; (b) Field lines surrounding a simple dipole magnet; (c) Radiograph showing field lines around a bar magnet levitating over an yttrium-barium-copper-oxide (YBCO) superconductor as in its superconducting state the YBCO expels all magnetic fields and thus repels the permanent magnet (Meissner effect); (d) Magnetic field trapped in a polycrystalline lead cylinder at different temperatures around $\mathrm{T}_{\mathrm{C}}=7.2 \mathrm{~K}$. The trapped flux (yellow regions left and right) inside a polycrystalline cylinder of lead is reconstructed in $3 \mathrm{D}$. When cooled to below its critical temperature in the presence of a weak magnetic field some flux is preserved inside the sample due to defects and grain boundaries and this remains trapped even after the field is switched off [2]. 
This has been demonstrated with a variety of magnetic systems. Figure $1 \mathrm{~b}$ reveals the familiar pattern of field lines around a simple dipole magnet, while Figure 1c shows the field around a magnet that is levitating over a cooled YBCO superconductor due to the Meissner effect. The rainbow color scale (from blue $=$ minimum to red $=$ maximum) used in the images is related to the intensity variations induced by the sample and by the applied magnetic field. The decay of the magnetic field with distance creates an annular structure of minima (blue) and maxima (red) representing the $2 \pi$ periodical rotation of the neutron spin. The imaging method can also be extended into three dimensions using a standard tomographic technique in some instances as indicated in Figure 1d, which shows the distribution of a magnetic field trapped inside a lead cylinder that becomes superconductive when cooled below the critical temperature of $\mathrm{T}_{\mathrm{c}}=7.2 \mathrm{~K}[2,3]$.

With the experimental setup presented one can investigate not only static states at constant magnetic field but also the dynamics of fields produced by AC currents with high frequencies. Interesting examples are the visualization of the skin effect in bulk conductors [10] or the magnetic field produced by a solenoid driven with a high-frequency $(3 \mathrm{kHz}) \mathrm{AC}$ current [11] as presented in Figure 2. In order to obtain images in short time intervals a stroboscopic method was applied where the detector was triggered by a signal from the power generator to take images at the same current amplitudes many times. The obtained images for a given current amplitude were summed up to obtain an image with reasonable signal-to-noise ratio.

a)

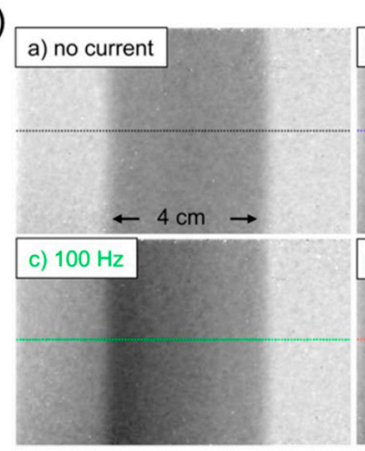

b)

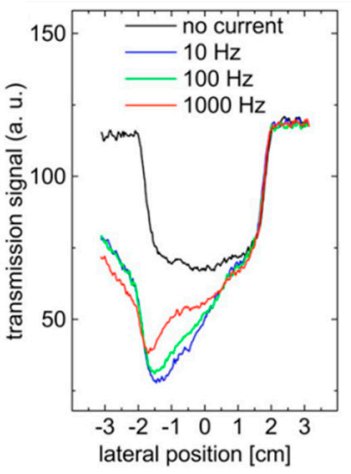

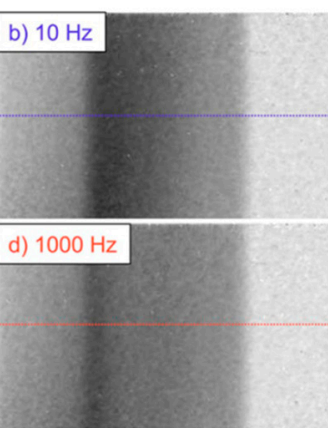

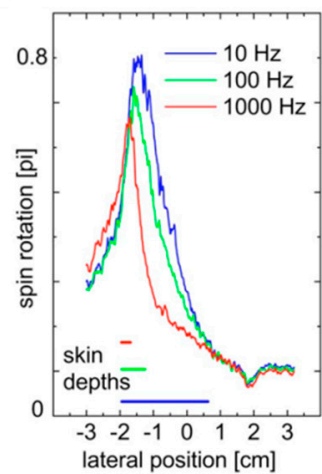

c)

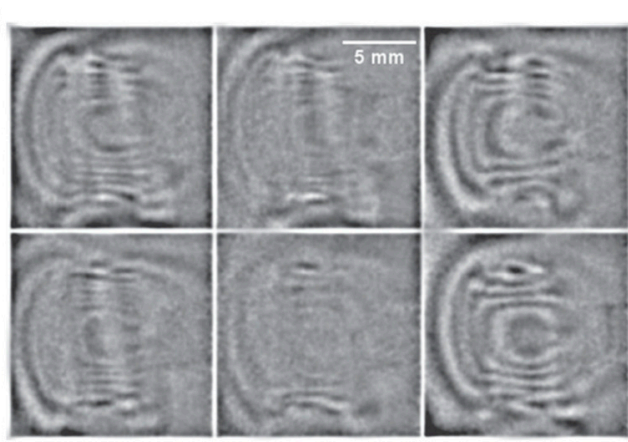

d)

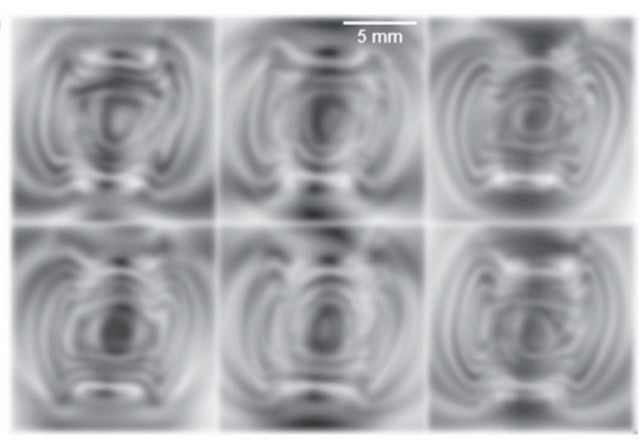

Figure 2. (a) Observation of skin effect in a bulk aluminum sample of $4 \mathrm{~cm}$ diameter without applied current and with $25 \mathrm{~A}$ alternating current at $10 \mathrm{~Hz}, 100 \mathrm{~Hz}$, and $1000 \mathrm{~Hz}$; (b) Analysis of the spin-polarized image data shows an inhomogeneous magnetic field distribution that can be related to a current distribution similar to this observed in the experiment. The current flows close to the surface of the conductor at high AC frequencies $(1 \mathrm{kHz})$ which is a proof that the skin effect is observed (Reprinted from [10], with the permission of AIP Publishing); (c) Spin-polarized neutron image of magnetic field produced by a 15-loop coil running at $3 \mathrm{kHz}$ current with $10 \mathrm{~A} \mathrm{rms}$ amplitude measured in a polychromatic neutron beam. The time slice interval of the shown images is $10 \mu \mathrm{s}$; (d) Simulations performed based on experimental parameters [11]. 
The benders have several properties that are not ideal for imaging experiments aiming at high-spatial resolution: (i) for polarizers working in the reflection mode the polarized neutron beam is deflected. This implies that sample and detector must be shifted transversally in order to be aligned with the beam; (ii) the available field of view is relatively small (1 to $4 \mathrm{~cm}$ ), which is a problem for investigations of large samples and their surroundings; (iii) the bender collimates the beam along one direction, resulting in a significantly lower resolution along the other direction; (iv) the beam polarization is not uniform across the beam (it can fluctuate by up to $5 \%$ ); (v) the beam attenuation caused by the wafers causes strong striping inhomogeneities in the beam intensity profile (Figure $3 \mathrm{a}$ ); and (vi) the comparably strong magnets $(0.1 \mathrm{~T})$ needed to magnetize the benders require a sample-bender separation of $\sim 50 \mathrm{~cm}$ to ensure that the interaction between the magnetic field of the bender and that of the sample is negligible. This separation distance is a reason for geometrical blurring caused by the finite divergence of the beam.

a)

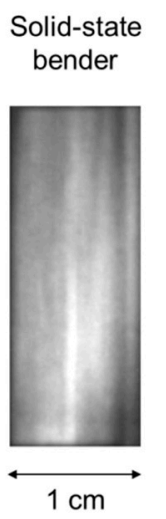

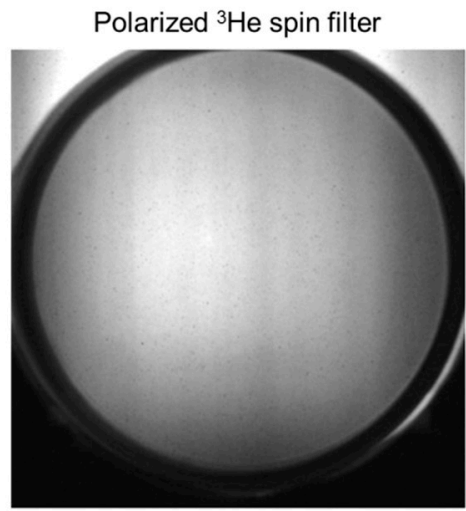

b)

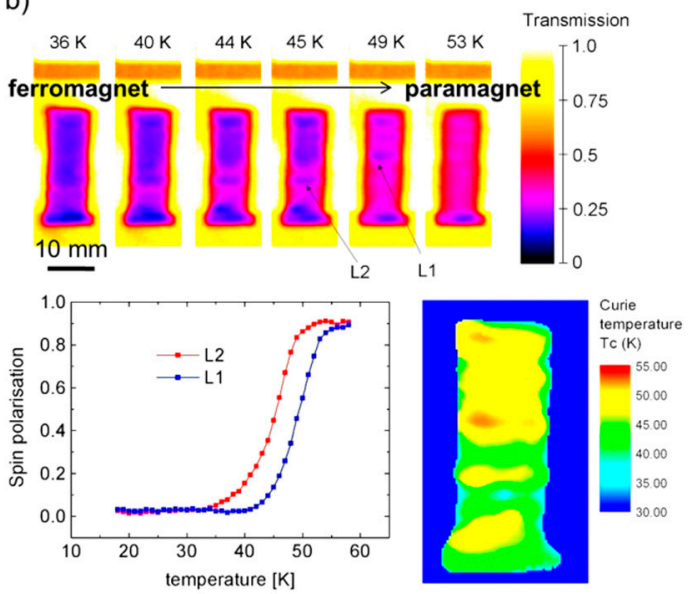

Figure 3. (a) Comparison between transmission images of a solid-state bender (left) and a polarized ${ }^{3} \mathrm{He}$ spin filter (right) [12]; (b) Mapping of phase transition process from the ferromagnetic to the paramagnetic state of a PdNi crystal $(3.24 \% \mathrm{Ni})$ using a polarized ${ }^{3} \mathrm{He}$ spin filter setup [13].

\subsection{Polarized ${ }^{3} \mathrm{He}$ Spin Filters}

The nuclear orbital moment of ${ }^{3} \mathrm{He}$ atoms can be polarized by optical pumping using the SEOP (spin-exchange) method. For the interaction of neutrons with the polarized ${ }^{3} \mathrm{He}$ gas in constant magnetic field, different absorption coefficients for parallel and anti-parallel neutron spin orientations with respect to the ${ }^{3} \mathrm{He}$ atoms can be obtained. In this way, only one spin component of the neutrons will be transmitted through the cell. The cells are made of glass and are filled with polarized ${ }^{3} \mathrm{He}$ gas under a certain pressure. They are placed in several $10-\mathrm{cm}$ long boxes in which a constant magnetic field is held. The spin filters do not alter the angular distribution of the beam and in this way do not affect spatial resolution (aside from the geometrical spread as the beam passes them) and produce homogeneous attenuation and polarization over the whole beam. A further advantage of ${ }^{3} \mathrm{He}$ cells is that they will accept and polarize neutrons over a large solid angle so that even a poorly collimated beam can be effectively polarized [12].

By emitting radiofrequency pulses with the Larmor frequency of the ${ }^{3} \mathrm{He}$ nuclei, transitions between the ${ }^{3} \mathrm{He}$ spin-states can be induced, allowing the polarizer and the analyzer to be realigned parallel or anti-parallel. Flipping the polarization of the spin filters is equivalent to flipping the polarization of the incident beam with an efficiency of $100 \%$ regardless of the neutron wavelength (although in practice there is a loss in polarization of $\sim 10^{-5}$ for each flip).

A crucial advantage of the use of filters over benders is that the magnetic field in the box is weak and well confined, allowing samples to be placed close to the front of (and in some cases even 
inside) the box. By attaching the scintillator directly to the rear surface of the analyzer cell, it is possible to reduce the sample-detector separation to $\sim 15-20 \mathrm{~cm}$, which brings down geometrical blur to $\sim 200 \mu \mathrm{m}$ [12].

The large and homogenous polarized neutron beam produced by the arrangement of ${ }^{3} \mathrm{He}$ spin filters has been used successfully for mapping phase transitions between ferromagnetic and paramagnetic states in a PdNi crystal with $3.24 \% \mathrm{Ni}$ as shown in Figure 3b [13]. The ferromagnetic material is characterized by the presence of arbitrarily oriented magnetic domains that depolarize the neutron beam and provide low neutron transmission. After increasing the temperature in some areas, the material becomes paramagnetic, which is characterized by the loss of the domain structure and the neutron beam is no longer depolarized and a rise in transmission is observed. The temperature of the sudden change of neutron transmission is taken as the critical temperature of the phase transition (the Curie temperature). The obtained Curie temperature map provides information about the inhomogeneity of Ni concentration that influences the critical temperature of the PdNi crystal.

The drawbacks of ${ }^{3} \mathrm{He}$ spin filters are the lower polarization ratio in comparison to solid state benders and the decay of the cell polarization with the time. In order to overcome the last drawback cells were developed and used recently for imaging in on-line pumping allowed to maintain polarisation [14]. A detailed comparison between solid-state benders and ${ }^{3} \mathrm{He}$ cells is presented elsewhere [12].

\section{Simulation and Quantification of Magnetic Fields}

In relatively simple cases, in which the magnetic field distribution of a sample is either well known (e.g., a permanent dipole magnet) or calculable (e.g., via the laws of electromagnetism) it is possible to compare the experimentally measured precession angle to calculated predictions. In the case of a well-known electrical current distribution flowing through a wire, the electromagnetically induced field can be calculated from the Biot-Savart law:

$$
\vec{B}(\vec{s})=-\frac{u_{0}}{4 \pi} \cdot I \cdot \frac{\overrightarrow{d s} \times \vec{r}}{r^{3}}
$$

where $\vec{B}$ is the field vector, $\mu_{0}$ the permeability of free space $\left(4 \pi \times 10^{-7} \mathrm{Hm}^{-1}\right), I$ is the current flowing in the wire, $\overrightarrow{d s}$ is an infinitesimal vector element of the wire pointing in the direction of the current, and $\vec{r}$ and $r$ are the unit vector and the distance, respectively, from the wire to the point where the field is to be calculated. Starting from Equation (1), the overall local spin rotation at a given point is then given by:

$$
\varphi=\gamma \cdot t \cdot B, \text { where } t \sim \lambda
$$

Once the field distribution of the system is known, it can be used to compute a 3D magnetic field matrix, with each element representing the magnetic field vector in a unit volume (a voxel). Neutrons with a defined polarization vector enter the first voxel and precess for a given time (depending on neutron velocity), leading to a new polarization vector, which is then passed to the next voxel along the trajectory of the neutron, and so on, Figure 4a. Once the neutrons have passed through the matrix the total angle of precession can be used together with (1) to find the intensity measured in the calculated image. Figure $4 \mathrm{~b}$ shows the experimentally measured and calculated field around a pair of small solenoids with rectangular cross section, separated by $20 \mathrm{~mm}$ and supported by an aluminum rod as the direct current passing through them was varied. The coils had roughly the same length of $24.4 \mathrm{~mm}$, a height of $7.5 \mathrm{~mm}$, a depth of $21.5 \mathrm{~mm}$, and 30 windings [3]. 
a)

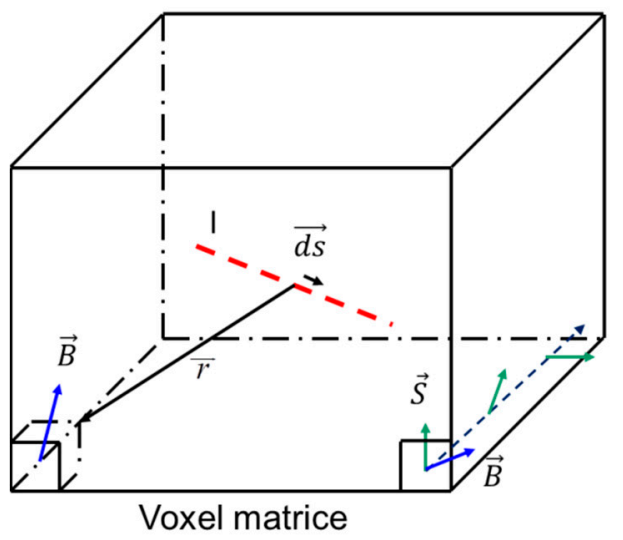

b)

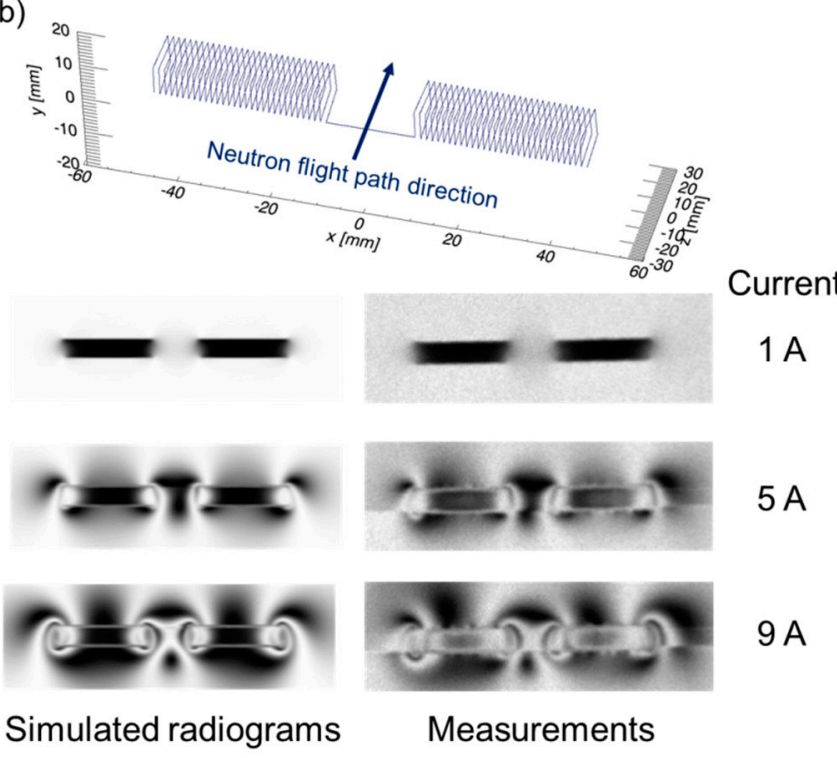

Figure 4. (a) Example for space discretization used in simulations of imaging with polarized neutrons. (b) Measurements and simulations of spin-precession image of rectangular double coil measured at different current values [3].

In general, the calculated and experimental results agree very well, though some deviations can be seen. These discrepancies occur because the calculations represent an idealized system where the coils are identical, perfectly aligned, self-contained and isolated from external influences. In reality, the coils used in the experiments suffer from irregularities due to their manufacture and differences in the measured intensity (between experiment and calculation) occur.

For the quantification of magnetic vector fields in $3 \mathrm{D}$, a neutron polarimetric setup is used. The spin of the polarized neutron beam is rotated by spin flippers (rotators) in order to send one of the three orthogonal components $\mathrm{x}, \mathrm{y}$ or $\mathrm{z}$ through the sample and in the same way the resulting spin orientation after precession in the magnetic field of the sample is analyzed by a second spin flipper (rotator) for each of the $\mathrm{x}, \mathrm{y}$ or $\mathrm{z}$ directions. From these 9 measurements (one initial component $\mathrm{x}, \mathrm{y}$ or $\mathrm{z}$ is analyzed in each of the three components $(\mathrm{x}, \mathrm{y}, \mathrm{z})$ of the spin precession angle) for each pixel of the detector, the polarization matrix $\mathrm{P}$ can be reconstructed correspondingly. Applying a tomographic approach, it is possible to reconstruct the modulus and the $\mathrm{x}, \mathrm{y}, \mathrm{z}$ components of the magnetic field vector for each point of the sample's volume and the space around it. Several approaches were developed and tested on simple samples with well-defined magnetic field distributions, e.g., a small solenoid producing a static magnetic field under DC current. The first results are shown in Figure 5, where a small solenoid was investigated by neutron tomography using the polarimetric arrangement at a steady state neutron source (research reactor). An iterative procedure based on simulations (Biot-Savart law) shows a good agreement with the experimental data and allows for a 3D visualization of the field produced by the solenoid [7]. A different approach using a pulsed neutron beam combined with a time-of-flight method was proposed recently and first experiments show promising reconstructions of the magnetic vector field produced by a current driven solenoid obtained by the Filtered-Back-Projection tomographic reconstruction algorithm [15]. 
a)
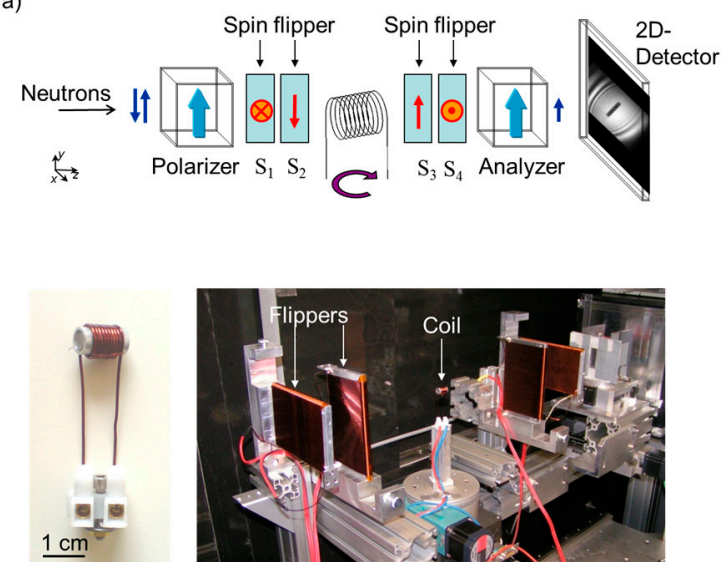

b) Initial spin direction: $x$

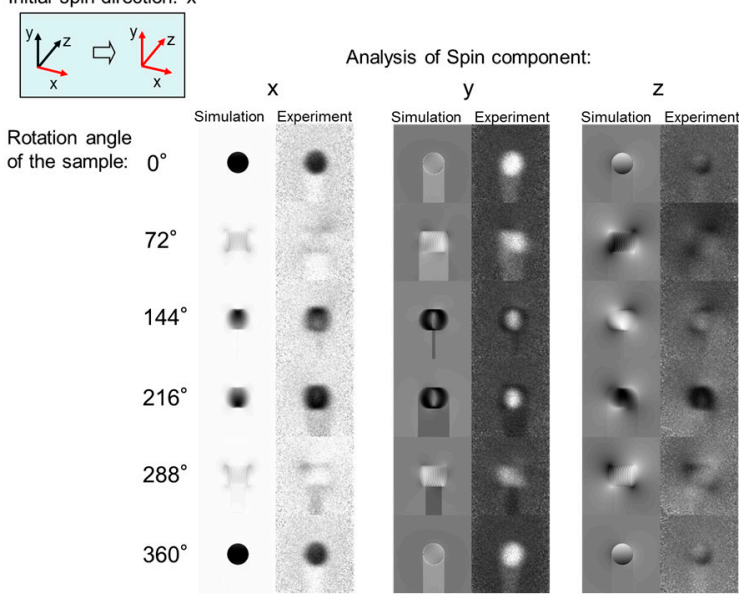

Figure 5. (a) Polarimetric arrangement for quantitative imaging with polarized neutrons; (b) Measured and simulated spin precession images for different rotation angles of a solenoid for initial spin direction $\mathrm{x}$ and analysis of the three orthogonal components of the spin precession angle.

\section{Conclusions}

Spin-polarized neutron imaging is a non-destructive and non-invasive method that has a number of advantages compared to 2D imaging techniques using garnet films or scanning techniques (for magnetic fields outside the sample). The method is applicable to very different environmental set-ups (e.g., for low or high temperature investigations) and samples can be investigated from almost any viewing angle. In this way, 3D information about the field distribution is revealed even if no mathematical tomographic reconstruction is applied. Measurements are fast because about one million pixels are acquired in only one image within a few seconds or minutes. No other method can compete with these advantages even for field measurements in free space. Time and spatially resolved measurements become possible.

The presence and controlled application of magnetic fields is essential in many fields of science and technology as well as in fundamental physics. For example, investigations of flux distribution and flux pinning in large superconducting samples, the skin effect in conductors or magnetic domain distributions in bulk ferromagnets can be visualized and studied in detail.

Conflicts of Interest: The authors declare no conflict of interest.

\section{References}

1. De Graef, M.; Zhu, Y. Preface. In Experimental Methods in the Physical Sciences; Marc De, G., Yimei, Z., Eds.; Academic Press: New York, NY, USA, 2001; pp. xv-xvi.

2. Kardjilov, N.; Manke, I.; Strobl, M.; Hilger, A.; Treimer, W.; Meissner, M.; Krist, T.; Banhart, J. Three-dimensional imaging of magnetic fields with polarized neutrons. Nat. Phys. 2008, 4, 399-403. [CrossRef]

3. Dawson, M.; Manke, I.; Kardjilov, N.; Hilger, A.; Strobl, M.; Banhart, J. Imaging with polarized neutrons. New J. Phys. 2009, 11, 043013. [CrossRef]

4. Kardjilov, N.; Hilger, A.; Manke, I.; Strobl, M.; Dawson, M.; Banhart, J. New trends in neutron imaging. Nucl. Instrum. Methods Phys. Res. Sect. A Accel. Spectrom. Detect. Assoc. Equip. 2009, 605, 13-15. [CrossRef]

5. Kardjilov, N.; Manke, I.; Hilger, A.; Strobl, M.; Banhart, J. Neutron imaging in materials science. Mater. Today 2011, 14, 248. [CrossRef]

6. Williams, W.G. Polarized Neutrons; Oxford University Press: Oxford, UK, 1998.

7. Strobl, M.; Kardjilov, N.; Hilger, A.; Jericha, E.; Badurek, G.; Manke, I. Imaging with polarized neutrons. Phys. B Condens. Matter 2009, 404, 2611-2614. [CrossRef] 
8. Strobl, M.; Pappas, C.; Hilger, A.; Wellert, S.; Kardjilov, N.; Seidel, S.O.; Manke, I. Polarized neutron imaging: A spin-echo approach. Phys. B Condens. Matter 2011, 406, 2415-2418. [CrossRef]

9. Krist, T.H.; Mezei, F. Solid state neutron polarisers and collimators. Proc. SPIE 2001, 4509. [CrossRef]

10. Manke, I.; Kardjilov, N.; Strobl, M.; Hilger, A.; Banhart, J. Investigation of the skin effect in the bulk of electrical conductors with spin-polarized neutron radiography. J. Appl. Phys. 2008, 104, 076109. [CrossRef]

11. Tremsin, A.S.; Kardjilov, N.; Strobl, M.; Manke, I.; Dawson, M.; McPhate, J.B.; Vallerga, J.V.; Siegmund, O.H.W.; Feller, W.B. Imaging of dynamic magnetic fields with spin-polarized neutron beams. New J. Phys. 2015, 17, 043047. [CrossRef]

12. Dawson, M.; Kardjilov, N.; Manke, I.; Hilger, A.; Jullien, D.; Bordenave, F.; Strobl, M.; Jericha, E.; Badurek, G.; Banhart, J. Polarized neutron imaging using helium-3 cells and a polychromatic beam. Nuclear Nucl. Instrum. Methods Phys. Res. Sect. A Accel. Spectrom. Detect. Assoc. Equip. 2011, 651, 140-144. [CrossRef]

13. Michael, S.; Andreas, N.; Sergey, M.; Martin, M.; Elbio, C.; Burkhard, S.; Christian, P.; Peter, B. Towards a tomographic reconstruction of neutron depolarization data. J. Phys. Conf. Ser. 2010, 211, 012025.

14. Dhiman, I.; Ziesche, R.; Wang, T.; Bilheux, H.; Santodonato, L.; Tong, X.; Jiang, C.Y.; Manke, I.; Treimer, W.; Chatterji, T.; et al. Setup for polarized neutron imaging using in situ $3 \mathrm{He}$ cells at the Oak Ridge National Laboratory High Flux Isotope Reactor CG-1D beamline. Rev. Sci. Instrum. 2017, 88, 095103. [CrossRef] [PubMed]

15. Sales, M.; Strobl, M.; Shinohara, T.; Tremsin, A.; Kuhn, L.T.; Lionheart, W.R.B.; Desai, N.M.; Dahl, A.B.; Schmidt, S. Three Dimensional Polarimetric Neutron Tomography of Magnetic Fields. arXiv, 2017.

(C) 2018 by the authors. Licensee MDPI, Basel, Switzerland. This article is an open access article distributed under the terms and conditions of the Creative Commons Attribution (CC BY) license (http:/ / creativecommons.org/licenses/by/4.0/). 